\title{
Overexpression of PCK1 Gene Antagonizes Hepatocellular Carcinoma Through the Activation of Gluconeogenesis and Suppression of Glycolysis Pathways
}

\author{
Yufu Tanga,b Yibing Zhang ${ }^{a} \quad$ Chunhui Wang ${ }^{a}$ Zhongyi Sun ${ }^{a} \quad$ Longfei Lia $^{a}$ \\ Shuqun Cheng ${ }^{c}$ Wenping Zhou ${ }^{\mathrm{a}}$
}

aDepartment of Hepatobiliary Surgery, The General Hospital of Shenyang Military Area Command,

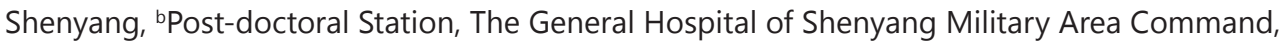
Shenyang, 'Eastern Hepatobiliary Surgery Hospital, Second Military Medical University, Shanghai, China

\section{Key Words}

Gluconeogenesis • Glycolysis • Hepatocellular carcinoma • Phosphoenolpyruvate carboxykinase 1

\begin{abstract}
Background/Aims: Gluconeogenesis, a reverse process of glycolysis, is suppressed in neoplastic livers. Cytoplasmic phosphoenolpyruvate carboxykinase (PEPCK-C/PCK1, encoded by $P C K 1$ ) is a step limiting enzyme of gluconeogenesis. The induced expression of the factor is reported to initiate gluconeogenesis process and antagonize hepatocellular carcinoma (HCC). In the current study, the effect of the modulation of PCK1 expression on HCC was assessed. Methods: The levels of PCK1 in clinical HCC tissues and different HCC cell lines were investigated with real time quantitative $P C R$, immunochemistry, and western blotting. Thereafter, the expression of $P C K 1$ gene was induced in two HCC cell lines and the effect of the overexpression on proliferation and migration potentials of HCC cells was detected with CCK8 assay, flow cytometry, TUNEL staining, and transwell assay. The activities of glycolysis and gluconeogenesis pathways in $P C K 1$-overexpressed HCC cell lines were detected with specific kits to underlie the mechanism by which PCK1 exerted its function. The results of the in vitro experiments were validated with HCC xenograft rat models. Results: The expression levels of PCK1 were suppressed in HCC samples and in cells derived from HCC tissues. According to the results of the in vitro assays, the overexpression of PCK1 decreased viability, induced apoptosis, and inhibited migration in both HCC cell lines. The effect was associated with the suppressed glycolysis and the induced gluconeogenesis pathways, represented by the enhanced production of glucose and the limited production of pyruvic acid, lactate, citrate, and malate. The results of the in vitro assays were confirmed in rat models in that the growth rate of solid HCC tumors was reduced in mice transplanted with PCK1-overexpressed HCC cells. Conclusion: Findings outlined in the current study demonstrated that activating gluconeogenesis process via $P C K 1$ overexpression was a potential treating strategy against HCC.

Prof. Wenping Zhou and

Prof. Cheng Shuqun
Department of Hepatobiliary Surgery, The General Hospital of Shenyang Military Area Command Shenyang, 100016, (China)

Tel. +86-024-28851241, E-Mail zwp0132@163.com or chengshuqun@aliyun.com 


\section{Cellular Physiology Cell Physiol Biochem 2018;47:344-355 \\ \begin{tabular}{l|l|l} 
DOI: 10.1159/000489811 & O 2018 The Author(s). Published by S. Karger AG, Basel \\
www.karger.com/cpb
\end{tabular} \\ Tang et al.: Activation of PCK1 Antagonizes HCC via Gluconeogenesis Initiation}

\section{Introduction}

During the past decades, emerging evidence demonstrates that metabolic reprogramming is a typical characteristic of cancers [1]. With the theory becoming a central subject in cancer study, metabolic pathways associated with oncogenesis, tumor progression, and tumor metastasis have been and are being discovered [2]. During the initiation of metabolic reprogramming in tumors, abnormal metabolic profiles promote glucose uptake, glycolysis, and lactic acid production even in the presence of sufficient oxygen, which supports growth, proliferation, and survival of tumor cells $[3,4]$. This so-called "Warburg" effect has been validated in distinct tumor types [5,6]. By bypassing the mitochondrial respiration in oxygen-rich conditions albeit the increased level of glycolysis, tumor cells successfully develop the key feature of metabolic reprogramming, now termed as aerobic glycolysis [2]. The "glucose addiction" endows tumor cells with enhanced metabolic flexibility for their anabolic requirements [7]. On the basis of the above studies, more attention is being paid to strategies that can restore normal metabolic conditions of cancer patients [8-11].

Gluconeogenesis, a reverse process of glycolysis, generates glucose from small carbohydrate substrates such as pyruvate, lactate, glycerol, and gluconeogenic amino acids [12]. Given that gluconeogenesis drives the metabolic flux in parallel but opposite to glycolysis, scientists believe that activating gluconeogenesis will interrupt metabolic reprogramming, initiate metabolic stress, and lead to the imbalance of energy homeostasis in cancer cells. Therefore, modalities capable of modulating gluconeogenesis are attracting lots of interest in recent years [13] and plenty effort has been made to facilitate the development of gluconeogenesis-based anti-cancer therapies [8-11]. Of types of cancers, hepatocellular carcinoma (HCC) has been proved to be sensitive to the treatments based on gluconeogenesis activation [8-11].

As the primary organ responsible for endogenous glucose production, the function of liver is closely associated with gluconeogenesis. Numerous studies indicate that the development of neoplasm in livers is always associated with the impaired gluconeogenesis $[9,10]$. Thus, the restoration of gluconeogenesis has been proposed as one of the primary tasks for the management of HCC. For example, p53 tumor suppressor protein enhanced gluconeogenesis by inducing the expressions of $G 6 P C$ and $P C K 2$, which prevented glucose from being shunted to pro-cancer pathways such as glycolysis and pentose phosphate pathway (PPP) [9]. Similarly, the inhibition of mTOR2 in HCC initiated shuttle of the glycolytic flux to gluconeogenesis along with inhibited proliferation and survival in cancer cell, which was also mechanistically linked to the up-regulation of G6PC and PCK1 [10].

Phosphoenolpyruvate carboxykinase (PEPCK/PCK, encoded by $P C K$ ) and glucose-6phosphatase (G6Pase, encoded by G6PC) are key enzymes of gluconeogenesis in the liver, of which PCK governs the rate-limiting step [2]. The enzyme catalyzes the conversion of oxaloacetate (OAA) to phosphoenolpyruvate (PEP) and its activity is distributed both in cytosol and mitochondria. As a result, two enzymatically indistinct isozymes, PEPCK-C (PCK1) and PEPCK-M (PCK2), encoded by different genes (PCK1 and PCK2) exist [14-16]. Compared with PCK2, PCK1 has been widely studied and is considered as a potential target for the development of novel treating methods for HCC $[8,10,17]$. However, most previous researches explored the function of PCK1 in an indirect way $[8,10,17]$, few studies have made attempt to elucidate the role of PCK1 in the development of HCC by directly controlling the expression of the factor. In the current study, the expression levels of PCK1 in clinical HCC samples were investigated. Thereafter, the expression of PCK1 in HCC cell lines was induced and the effect on the gluconeogenesis, glycolysis, proliferation, and migration in HCC cells was assessed. Furthermore, at molecular level, the expressions of indicators related to gluconeogenesis were detected to demonstrate the mechanism through which PCK1 exerted its anti-HCC function. 


\section{Cellular Physiology Cell Physiol Biochem 2018;47:344-355 \begin{tabular}{l|l} 
and Biochemistry Published online: May 17, 2018 & $\begin{array}{l}\text { (c) 2018 The Author(s). Published by S. Karger AG, Basel } \\
\text { www.karger.com/cpb }\end{array}$
\end{tabular} \\ Tang et al.: Activation of PCK1 Antagonizes HCC via Gluconeogenesis Initiation}

\section{Materials and Methods}

\section{Patients and tissue specimen collection}

Twelve HCC patients were included in the current study from The General Hospital of Shenyang Military Area Command. Subjects should meet the following criteria: 1) HCC samples from the hepatectomy must be identified to be primary HCC and para-tumor tissues sampled from the patients must contain no HCC cells; 2) Patients should have detailed information of clinicolpathological and prognostic information. Those who had received any therapy against HCC before the operations were excluded. Tumor and paratumor samples collected during the operations were preserved in $4^{\circ} \mathrm{C}$ and $-80^{\circ} \mathrm{C}$ respectively before sent for histological and molecular analyses. The study was approved by The General Hospital of Shenyang Military Area Command ethics committee for the relating screening, inspection, and data collection of the patients. All the subjects signed written informed consent forms. All the works were undertaken following the provisions of the Declaration of Helsinki.

\section{Chemicals and antibodies}

Antibody against PCK1 was purchased from USCN Life Science Inc (Catl. No. PAA936Hu01, Wuhan, China). Antibody against G6PC was purchased from Abcam (Catl. No. ab133964, Cambridge, MA). Antibody against cleaved caspase 3 was purchased from CST (Catl. No. \#9661, USA). Antibody against $\beta$-actin was purchased from Santa cruz Biotechnology, Inc (Catl. No. sc-47778, Santa Cruz, CA). Secondary goat anti-rabbit HRP IgG antibody (Catl. No. A0216, China) and goat anti-mice HRP IgG antibody (Catl. No. A0208, China), and protein concentration detection kit (Catl. No. P0011) were purchased from Beyotime Biotechnology. Pyruvic acid detection kit (Catl. No. A081), glucose detection kit (Catl. No. F006), citrate detection kit (Catl. No. A128), and lactate detection kit (Catl. No. A019-2) were purchased from Nanjing Jiancheng Bioengineering Institute, China. PicoProbeTM fructose-6-phosphate fluorometric assay kit (Catl. No. K689) and malate colorimetric assay kit (Catl. No. K637) were purchased from BioVision (USA). In situ cell death detection kit (Catl. No. 11684795910) was purchased from Roche (Switzerland).

\section{Cell cultures and animals}

Human HCC cell line CSQT-2 was a gift from Prof. Shuqun Cheng. Human HCC cell lines hep3B (Catl. No. TCHu106) and Huh7 (Catl. No. TCHu182) were purchased from Shanghai Cell Bank of Chinese Science Academy. Human HCC cell line SMMC-7721 (Catl. No. ZQ0029) was purchased from Zhongqiaoxinzhou Biotech Company. All the cell lines were cultured in DMEM supplemented with 10\% FBS and penicillin/ streptomycin at $37^{\circ} \mathrm{C}$ in an atmosphere of $5 \% \mathrm{CO}_{2}$ and $95 \%$ air. All the cell lines were cultured at a density that allowed cell division throughout the experiments. The expression of PCK1 at mRNA and protein levels in different cells were quantified with reverse transcription quantitative PCR (RT-qPCR) and western blotting assay respectively. SPF BALB/c-nu mice were purchased from Vital River (Beijing, China) and maintained in cages at room temperature $\left(20-25^{\circ} \mathrm{C}\right)$ with a constant humidity $(55 \pm 5 \%)$ with free access to food and water in a 12:12-h light-dark cycle. All the animal experiments were conducted in the accordance with the Institutional Animal Ethics Committee and Animal Care Guidelines for the Care and Use of Laboratory Animals of The General Hospital of Shenyang Military Area Command.

\section{Construction of PCK1 expression vector and cell transfection}

The whole ORF of PCK1 was obtained from GenBank database (Accession number: NM_002591) and ligated into pcDNA3.1 to form PCK1 expression vector pcDNA3.1-PCK1. According to the detections of PCK1 levels in different HCC cell lines, two cell lines with relatively lower PCK1 levels, hep3B and SMMC-7721, were selected for transfection of pcDNA3.1-PCK1 using Lipofectamine 2000 (Catl. No. 1024993, Invitrogen, San Diego, CA) according to the instruction for manufacturers. Each cell line was divided into three groups: A Control group, parental cells. B NC group, cells transfected with pcDNA3.1 vector . C PCK1 group, cells transfected with pcDNA3.1-PCK1 vector. Stable transfection was selected with G418 (400 $\mu \mathrm{g} / \mathrm{mL})$ and verified with RT-qPCR and western blotting.

Transplantation of HCC cells into nude mice

In vivo HCC model was induced by transplanting different HCC cell lines (hep3B and SMMC-7721) into the back skin of nude mice. BALB/c-nu mice were randomly divided into three groups: A Control group, 


\section{Cellular Physiology Cell Physiol Biochem 2018;47:344-355

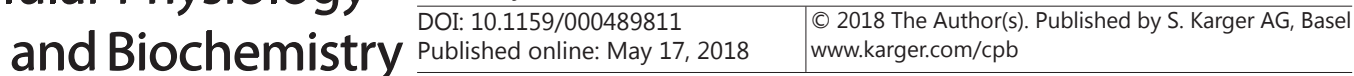 \\ Tang et al.: Activation of PCK1 Antagonizes HCC via Gluconeogenesis Initiation}

BALB/c-nu mice transplanted with HCC cells. B NC group, BALB/c-nu mice transplanted with HCC cells transfected with pcDNA3.1 vector. C PCK1 group, BALB/c-nu mice transplanted with HCC cells transfected with pcDNA3.1-PCK1 vector.. All the mice were raised under the same condition for 21 days to allow the formation of solid tumor. Tumor volume was measured every three days since the $7^{\text {th }}$ day after the transplantation: tumor volume $=[$ (length of major axis $\left.) \times(\text { length of minor axis })^{2}\right] / 2$. Upon completion of the raise, mice were sacrificed and tumor specimens were collected for histological and molecular analyses.

\section{$R T-q P C R$}

For RT-qPCR detection, total RNA in tumor tissues and HCC cells was extracted using RNA purified total RNA extraction kit according to the manufacturer's instruction (Calt. No. RP1201, BioTeke, Beijing, China). $\beta$-actin was selected as the internal reference gene. The total RNA was reversely transcribed to cDNA templates using super M-MLV reverse transcriptase (Calt. No. RP6502, BioTeke, Beijing, China). The final qPCR reaction mixture of volume $20 \mu \mathrm{L}$ consisted of $10 \mu \mathrm{L}$ of SYBR GREEN mastermix, $0.5 \mu \mathrm{L}$ of each primers [PCK1, forward: 5'-GCTGGTGTCCCTCTAGTCTATG-3', reverse: 5'-GGTATTTGCCGAAGTTGTAG-3'; G6PC, forward: 5'-GTGCCAAGGAAATGAGGATG-3', reverse: 5'-CACGGACACCAAGATGAACC-3'; hexokinase 2 (HK2), forward: 5'-TCTACCACATGCGCCTCTCT-3', reverse: 5'-GCCCATTGTCCGTTACTTTC-3'; 6-phosphofructokinase 1 (PFK1), forward: 5'-CCAAGCGTCGGGTGTTTATC-3', reverse: 5'-TGCAGGTCTCGAATGGTGAAG-3'; pyruvate kinase M2 (PKM2), forward: 5'-CGCTGGATAACGCCTACAT-3', reverse: 5'-CCATTTTCCACCTCCGTC-3'; $\beta$-actin forward: 5'-CTTAGTTGCGTTACACCCTTTCTTG-3', reverse: 5'-CTGTCACCTTCACCGTTCCAGTTT-3'], $1 \mu \mathrm{L}$ of the cDNA template, and $8 \mu \mathrm{L}$ of Rnase free $\mathrm{H}_{2} \mathrm{O}$. Amplification parameters were as following: denaturation at $95^{\circ} \mathrm{C}$ for $10 \mathrm{~min}$, followed by 40 cycles at $95^{\circ} \mathrm{C}$ for $10 \mathrm{~s}, 60^{\circ} \mathrm{C}$ for $20 \mathrm{~s}$ and $72^{\circ} \mathrm{C}$ for $30 \mathrm{~s}$, and the reaction was stopped at $4^{\circ} \mathrm{C}$ for $5 \mathrm{~min}$. Relative expression levels of the target molecules were detected and calculated with ExicyclerTM 96 (BIONEER) according to the expression of $2^{\text {-ש⿴囗十 }}$.

\section{Western blotting assay}

Total protein in tumor tissues and HCC cells was extracted using the total protein extraction kit according to the manufacturer's instruction (Catl. No. WLA019, Wanleibio, China). $\beta$-actin was used as the internal reference. Concentrations of protein samples were determined using BCA method. $20 \mu \mathrm{L}$ of protein $(40 \mu \mathrm{g})$ was subject to a $10 \%$ sodium dodecylsulfate polyacrylamide gel electrophoresis (SDS-PAGE). Target proteins were transferred onto polyvinylidene difluoride (PVDF) sheets and the membranes were washed with TTBS for $5 \mathrm{~min}$ before incubation in skim milk powder solution for $1 \mathrm{~h}$. Primary antibodies against PCK1 (1:400), G6PC (1:1000), cleaved caspase 3 (1:1000), and $\beta$-actin (1:1000) were incubated with membranes at $4^{\circ} \mathrm{C}$ overnight. After four washes using TTBS, secondary HRP IgG antibodies (1:5000) were added onto the membranes for $45 \mathrm{~min}$ at $37^{\circ} \mathrm{C}$. After additional six washes using TTBS, the blots were developed using Beyo ECL Plus reagent and the results were detected in the Gel Imaging System. The relative expression levels of proteins were calculated with Gel-Pro-Analyzer (Media Cybernetics, USA).

\section{Immunohistochemical detection}

Sections were made from different samples, examined independently by senior pathologists, and frozen immediately for PCK1 detection. For immunohistochemical detection, tissue slides were placed in $60^{\circ} \mathrm{C}$ overnight before being incubated with dimethylbenzene for dewaxing. The slides were hydrated with different concentrations of alcohol ( $100 \%$ for $5 \mathrm{~min}, 95 \%$ for $5 \mathrm{~min}, 85 \%$ for $5 \mathrm{~min}$, and $75 \%$ for $5 \mathrm{~min}$.) followed by washed with $\mathrm{H}_{2} \mathrm{O}_{2}$ for 5 min. Then slides were fixed using methanol solution with $3 \% \mathrm{H}_{2} \mathrm{O}_{2}$ and blocked with $1 \% \mathrm{BSA}$ for $30 \mathrm{~min}$ at $37^{\circ} \mathrm{C}$. They were then incubated with primary antibody against PCK1 (1:100) at $37^{\circ} \mathrm{C}$ for $30 \mathrm{~min}$ before incubated at $4^{\circ} \mathrm{C}$ overnight. After four cycles of $0.01 \mathrm{M}$ PBS washing, $5 \mathrm{~min}$ for each cycle, secondary antibody $(1: 200)$ was added onto the slides and placed at $37^{\circ} \mathrm{C}$ for $30 \mathrm{~min}$ before another four cycles of PBS wash. Then diaminobenzidine (DAB) was added to the slides and reacted for 3-10 min until the reaction was stopped by $\mathrm{ddH}_{2} \mathrm{O}$. Slides were re-stained using haematoxylin and dehydrated. Results were detected using a microscope (BX53, OLUMPUS, Japan) at 400× magnification

\section{Determination of activity of glycolysis pathway in PCK1-overexpressed HCC cell lines}

The activity of glycolysis pathway was determined by quantifying the levels of different products (including pyruvic acid, lactate, fructose-6-phosphate, and malate) of glycolysis. The expressions of key kinases involved in the regulation of glycolysis (including HK2, PFK1, and PKM2) were detected with RTqPCR.

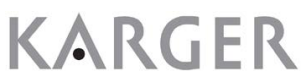




\section{Cellular Physiology Cell Physiol Biochem 2018;47:344-355 \begin{tabular}{c|c|c|} 
DOI: 10.1159/000489811 & O 2018 The Author(s). Published by S. Karger AG, Basel \\
www.karger.com/cpb
\end{tabular} \\ Tang et al.: Activation of PCK1 Antagonizes HCC via Gluconeogenesis Initiation}

Glucose production assay

The production of glucose in cells was detected using glucose detection kit according to the manufacturers' instruction and normalized to total protein.

\section{CCK-8 assay}

Cell viabilities of PCK1-transfected HCC cell lines were measured using a CCK-8 method: briefly, $100 \mu \mathrm{L}$ CCK- 8 solution was added to the cultures and incubated at $37^{\circ} \mathrm{C}$ for $1 \mathrm{~h}$. The OD values at $450 \mathrm{~nm}$ of different treatments were detected using a Microplate Reader and normalized as relative cell viability (\%) compared to control cells (untreated cells): $\left[\mathrm{OD}_{450}\right]_{\text {sample }} /\left[\mathrm{OD}_{450}\right]_{\text {control }} \times 100[18]$

\section{Flow cytometry assay}

The apoptotic rates of HCC cells were determined with flow cytometry. Cells in different groups were collected with centrifugation at $2000 \mathrm{rpm}$ for $5 \mathrm{~min}$. Then the cell apoptotic rates were measured using an Annexin V-FITC Apoptosis Detection Kit (WLA001c, Wanleibio, Shenyang, China) according to the instruction for manufacturers: briefly, $5 \mu \mathrm{L}$ Annexin $\mathrm{V}$ was added to different wells. After incubation with Annexin $\mathrm{V}$ for $10 \mathrm{~min}$ at room temperature, the cells were re-suspended with $1 \times$ Binding buffer and added with $5 \mu \mathrm{L}$ Propidium Iodide. The apoptotic rates were analyzed using a FACScan flow cytometry (Accuri C6, BD, USA) and the total apoptotic rate (UR+LR-all apoptosis cell percentage) was equal to the sum of the late apoptotic rate (UR, upper right quadrant-advanced stage apoptosis cell percentage) and the early apoptotic rate (LR, lower right quadrant-prophase apoptosis cell percentage).

\section{Terminal-deoxynucleoitidyl Transferase Mediated Nick End Labeling (TUNEL) staining}

Cell apoptosis was detected using in situ cell death detection kit following the instruction for manufacturers. The apoptotic cells were stained brown and the results were detected using a microscope (BX53, OLUMPUS, Japan) at 200× magnification.

\section{Transwell experiment}

The transwell experiment was performed to evaluate the migration ability of HCC cells: $200 \mu \mathrm{L}$ incubation (with $1 \mathrm{mM} \mathrm{MgCl}$ ) medium containing $1 \times 10^{4}$ cells was seeded into the upper chamber of transwell chambers (Corning star, Cambridge, MA). Then cells were incubated at $37^{\circ} \mathrm{C}$ in an atmosphere of $5 \% \mathrm{CO}_{2}$ and $95 \%$ air for $24 \mathrm{~h}$ to allow the migration through the porous membrane. Upon completion of the culture, cells remaining at the upper surface of the chamber were completely removed. The lower surfaces of the membranes were fixed with $4 \%$ paraformaldehyde for $20 \mathrm{~min}$ and stained in a solution containing $0.5 \%$ (w/v) crystal violet for $5 \mathrm{~min}$. After being washed using $\mathrm{ddH}_{2} \mathrm{O}$, numbers of cells in five fields of each group were determined using Image-Pro Plus 6.0 software (Nikon) at 200× magnification and averaged.

\section{H\&E staining}

The histological changes in tumor specimens collected from nude mice were observed using Hematoxylin and eosin (H\&E) staining: briefly, tissues were placed into Bouin solution ( $4 \%$ formaldehyde) for perfusion fixation, dehydrated using different concentrations of alcohol, and vitrified in dimethylbenzene. Afterwards, samples were embedded in paraffin, sectioned, and stained with H\&E and the results were detected under a microscope at 200× magnification. The nuclei in tumor tissue were stained blue and the cytoplasms were stained pink, and the degree of staining varied with the age of cells.

\section{Statistical analysis}

All the data were expressed in the form of mean \pm standard deviation (SD). One-way analysis of variance (ANOVA), ANOVA for repeated measurements, and post doc test by Duncan method were performed using general liner model. Statistical significance was accepted with $P$ value $<0.05$. All the statistical analyses were conducted using SPSS version 19.0 (IBM, Armonk, NY, USA).

\section{Results}

Expression levels of PCK1 were inhibited in clinical HCC samples and in HCC cell lines

In total, 12 HCC patients were included in the current study. At mRNA, the level of PCK1 was highest in para-tumor samples (Fig. 1A), and decreased in tumor and tumor thrombus KARGER 


\section{Cellular Physiology Cell Physiol Biochem 2018;47:344-355 and Biochemistry Published onIIne: May 17, $2018 \quad \begin{aligned} & \text { DOI: 10.1159/000489811 } 2018 \text { The Author(s). Published by S. Karger AG, Basel } \\ & \text { www.karger.com/cpb }\end{aligned}$ \\ Tang et al.: Activation of PCK1 Antagonizes HCC via Gluconeogenesis Initiation}

tissues. Compared with tumor samples, the level of $P C K 1$ was even lower in tumor thrombus tissues. Based on statistical analyses, the difference between each two groups was significant (Fig. 1A) $(P<0.05)$. The changing pattern of protein was similar to that of mRNA: tissues from para-tumor samples had the highest level of PCK1 while tumor thrombus tissues had the lowest PCK1 level (Fig. 1B and Fig. 1C) $(P<0.05)$. Moreover, as shown in Fig. 1D, the immunohistochemical detection confirmed the results of western blotting assay: PCK1 positive cells characterized by brownish-yellow particles were more extensively distributed in para-tumor tissues.

To verify the expression status of PCK1 in HCC, the levels of PCK1 in four HCC cell lines were also detected. As shown in Fig. 2, the expression of PCK1 was lower in hep3B, Huh7, and SMMC-7721 cells which were derived from tumor tissues compared with CSQT-2 cells derived from portal vein tumor thrombus tissues. Two cell lines, hep3B and SMMC-7721 with the relatively lower levels of PCK1 were selected for subsequent assays (Fig. 2).

\section{Overexpression of PCK1 blocked the glycolysis process in HCC cell lines}

The transfection of expression vector increased the expression of PCK1 both at mRNA and protein levels (Fig. 3) in HCC cells. The overexpression of PCK1 suppressed the production of pyruvic acid, lactate, citrate, and malate in HCC cells, representing the blockade of glycolysis process due to the induced expression of PCK1 (Fig. 4A) $(P<0.05)$. However, the production of fructose-6-phosphate was not significantly influenced by the overexpression of PCK1 (Fig. 4A), which might be attributed to the increased production of fructose-6-phosphate in gluconeogenesis induced by PCK1 overexpression. The results of biochemical detection were further validated by RT-qPCR. As shown in Fig. 4B, the expressions of $H K 2$ and PKM2 were inhibited, representing an impaired glycolysis process by $P C K 1$ overexpression. It was also found that the expression of PFK1 was not influenced by PCK1 overexpression, partially explaining the production pattern of fructose-6phosphate in cells of different groups.

Overexpression of
PCK1 decreased
viability, induced
$\begin{aligned} & \text { apoptosis, and } \\ & \text { suppressed migration } \\ & \text { ability in HCC cell } \\ & \text { lines } \\ & \text { The viabilities of }\end{aligned}$
both HCC cell lines were detected with CCK-8 assay and the results showed that the overexpression of PCK1 markedly reduced cell viability (Fig. 5A) $(P<0.05)$. Correspondingly, the apoptotic process in both cell lines were initiated by PCK1 overexpression as detected by flow cytometry (Fig. 5B) $(P<$ $0.05)$. For hep3B cells, the late apoptotic rate was higher than the

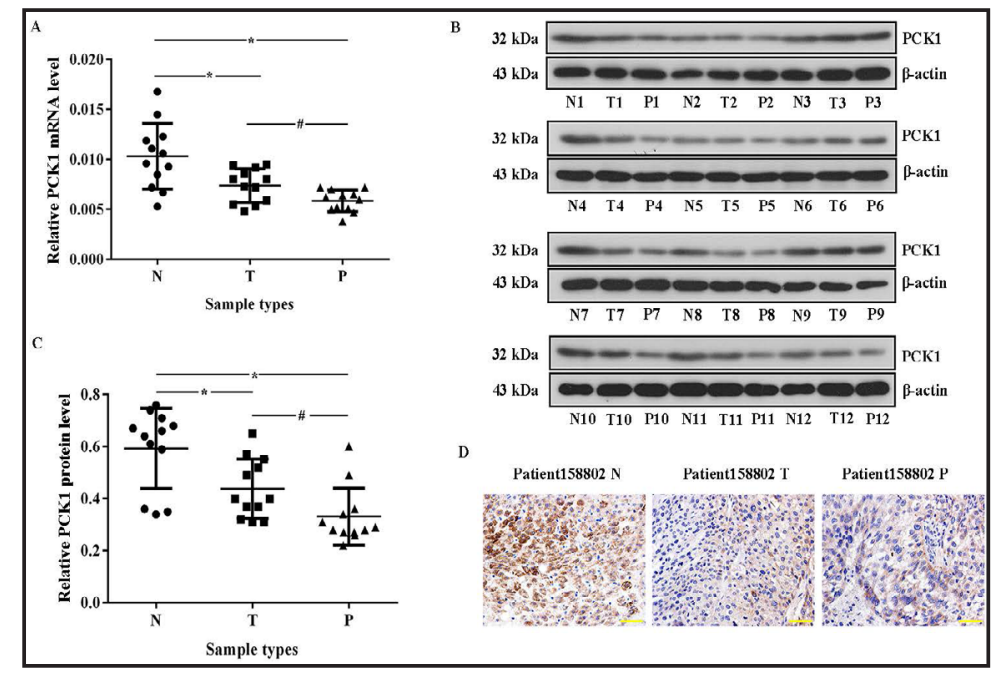

Fig. 1. Expression of PCK1 was suppressed in clinical HCC tissues. A, quantitative analysis results of RT-qPCR detection for PCK1 gene with clinical samples. B, representative images of western blotting assay for PCK1 with clinical samples. C quantitative analysis results of western blotting assay for PCK1 with clinical samples. D, representative images of immunochemical detection of PCK1 expression in Patient158802and PCK1 positive cells were stained brownish-yellow. N represents paratumor tissues. T represents tumor tissues. Scale bar, $100 \mu \mathrm{m}$. P represents tumor thrombus tissues. "*”, significantly different from $\mathrm{N}$ samples, $\mathrm{P}<0.05$. "\#”, significantly different from $\mathrm{T}$ samples, $\mathrm{P}<0.05$. Each assay was represented by 12 replicates. 
Fig. 2. Selection of HCC cell lines with lowest expression level of PCK1 gene. The expressions of PCK1 were lower in hep3B, Huh7, and SMMC-7721 cells which were derived from HCC tissues compared with CSQT2 cells which were derived from portal vein tumor thrombus. Based on the detecting results, hep3B and SMMC-7721 were selected for further experiments. Each assay was represented by three replicates.

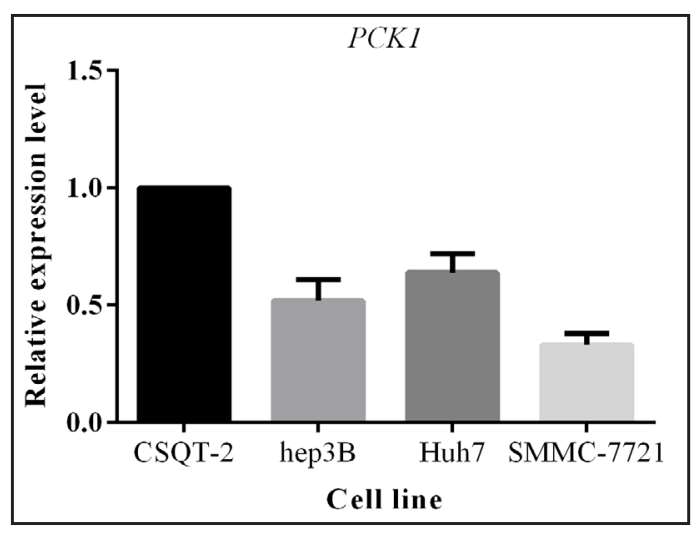

early apoptotic rate, but for SMMC-7721 cells, a reserve pattern was observed. The effect of PCK1 overexpression on cell apoptosis was further assessed by TUNEL staining and western blotting detection of cleaved caspase 3: the induced expression of PCK1 was associated with higher apoptotic rates (Fig. 5C) and higher cleaved caspase 3 levels (Fig. 5D), confirming the pro-apoptosis function of PCK1 in HCC cells. Except for the influence on survival of HCC cells, the overexpression of PCK1 also impaired migration potential of HCC cells. In transwell assays, the average numbers of cells penetrating the membranes were significantly smaller in PCK1 groups compared with Control or NC group (Fig. 5E) $(P<0.05)$.

\section{Antagonizing effect of PCK1 on HCC cells was exerted by activating process}

To confirmtheincreased

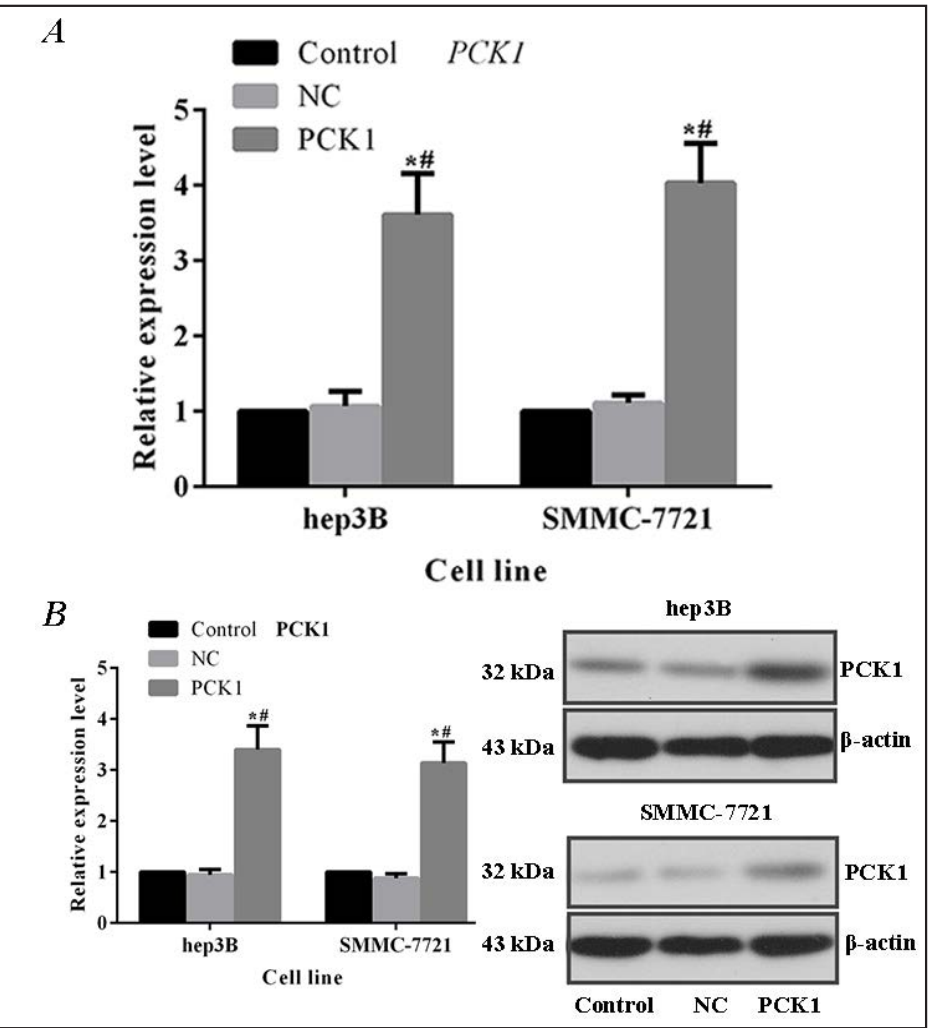

Fig. 3. Levels of PCK1 was increased in hep3B and SMMC-7721 cell lines after transfection of expression vector. A, quantitative analysis results of RT-qPCR detection for PCK1 gene with HCC cell sample. B, representative images and quantitative analysis results of western blotting assay for PCK1 with HCC cell samples. "*”, significantly different from Control group, $\mathrm{P}<0.05$. “\#”, significantly different from NC group, $\mathrm{P}<0.05$. Each assay was represented by three replicates. level of gluconeogenesis in HCC cells, the expression of another step limiting enzyme in gluconeogenesis, G6PC, was also detected. Both at mRNA and protein levels, the overexpression of PCK1 induced the expressions of G6PC (Fig. 6A and 6B). Moreover, the production of glucose in PCK1overexpressed cell lines was increased as well, confirming the activation of gluconeogenesis (Fig. 6C). The initiation of gluconeogenesis process was concomitant with the inhibiting effect of PCK1 overexpression on HCC cells, which implied that the function of PCK1 in HCC was related to the activation of gluconeogenesis.

\section{KARGER}




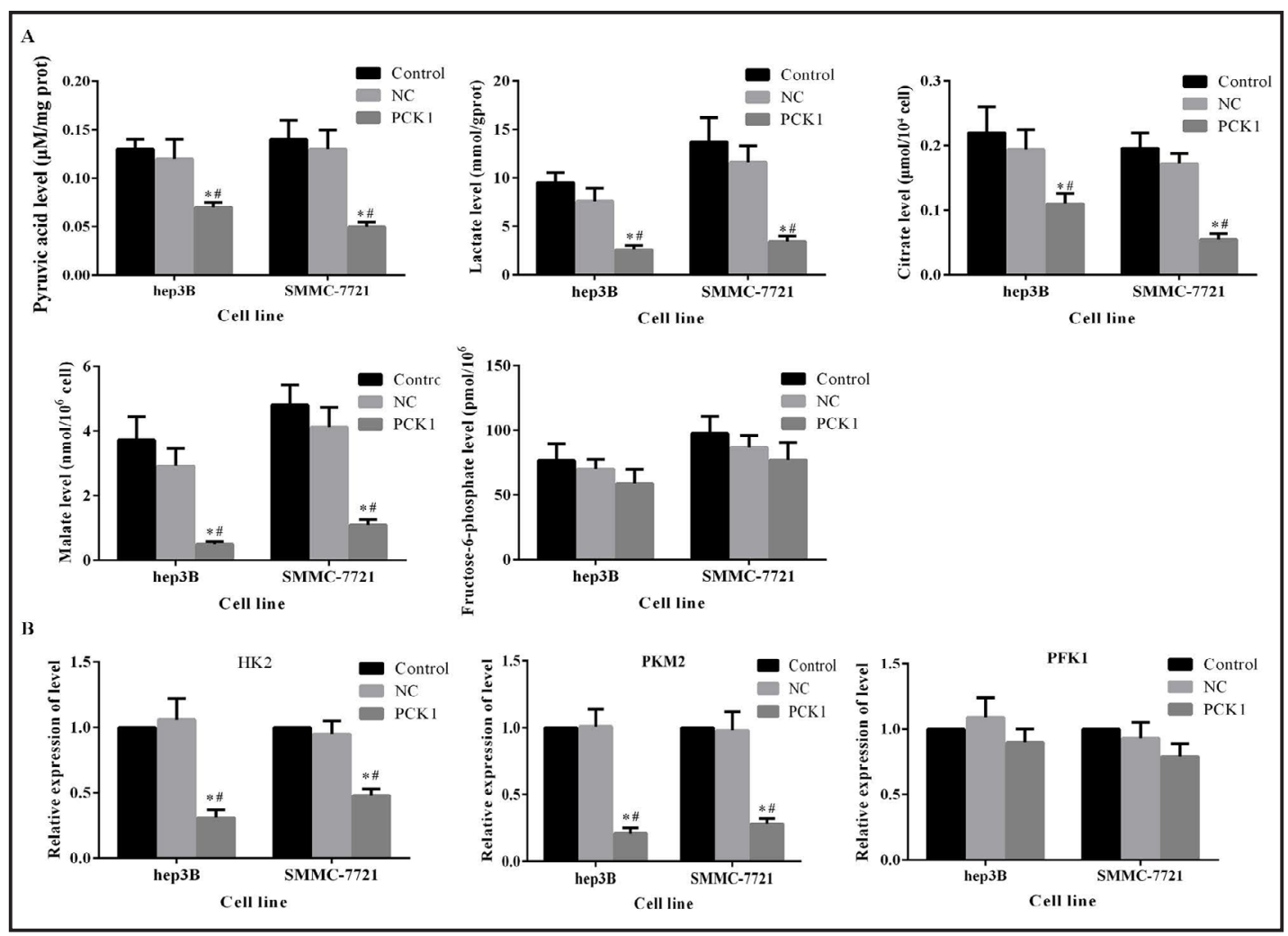

Fig. 4. Overexpression of PCK1 gene suppressed glycolysis process. A, quantitative analysis results of detection of pyruvic acid, lactate, citrate, malate, and fructose-6-phosphate production in different cell lines. B, quantitative analysis results of RT-qPCR detection for HK2, PKM2, and PFK1 expression with HCC cell samples. "*”, significantly different from Control group, $\mathrm{P}<0.05$. “\#”, significantly different from NC group, $\mathrm{P}<0.05$. Each assay was represented by three replicates.

\section{Overexpression of PCK1 inhibited the formation of solid tumor in vivo}

To validate the inhibiting effect of PCK1 on tumorigenecity of HCC, BALB/c-nu mice were transplanted with HCC cells to induce in vivo HCC models. As shown in Fig. 7A and Fig. 7B, volume of solid tumors in different groups kept increasing until the completion of house. For mice transplanted with hep3B cells, the average tumor volume in PCK1 group was significantly smaller than Control and NC groups, and the differences since the second sampling time point were statistically significant (Fig. 7A; Table 1) $(P<0.05)$. A similar growth pattern was also detected for assays with SMMC-7721 cells (Fig. 7B; Table 1) $(P<0.05)$. Illustrated by H\&E staining, in samples overexpressing PCK1, cell integrity was impaired, cytoplasms spread in wider range, and adhesion between cells were broken (Fig. 7C and Fig. 7D). Corresponding to the RT-qPCR and western blotting detection results of the in vitro experiments, the expressions of PCK1 and G6PC were both up-regulated, representing the initiation of gluconeogenesis in vivo (Fig. 7E-7H).

\section{Discussion}

Liver plays a determinant role in the maintainance of blood glucose concentration by supplying glucose to circulation and by removing glucose from circulation after meal ingestion [19]. One of the critical processes involved in the regulation of blood glucose by liver is gluconeogenesis. The process is described as the production of glucose from noncarbohydrate carbon sources and a reverse glycolysis pathway [2]. It is well recognized 
that the concomitant
activation or
suppression of
glycolysis and
glucone ogenesis
will cause a
metabolic stress [2]. The metabolic re progra m m in g in cancer cells is mainly attributed to the "aerobic glycolysis" process which enhances the metabolic flexibility of cancer cells [7]. Thus, the modulation of glycolysis/ gluconeogenes is has reasonably become an attractive therapeutic strategy for treatments of cancers.

In the present study, the expression and function of PCK1, a step limiting enzyme of gluconeogenesis, was detected in vivo and in vitro and employed as a target for the regulation of gluconeogenesis in HCC cells. The enzyme allows the conversion of small carbon metabolites and tricarboxylic acid cycle metabolites into glycolysis intermediates [9]. Based on the investigation with clinical HCC samples and different HCC cell lines, the expression of PCK1 was clearly suppressed in HCC. The overexpression of PCK1 in two HCC cell lines with relatively lower PCK1 levels inhibited cell viability and migration. Moreover, the antagonizing effect of PCK1 overexpression on HCC cells was concomitant with the suppression of glycolysis and the induction of gluconeogenesis, confirming the conclusion that the promoted gluconeogenesis in HCC would detrimentally affect cancer cell survival [10].

The suppression of gluconeogenesis in HCC has been reported by previous studies $[9,10$, $20,21]$. Thus, exploring factors activating gluconeogenesis has become a central subject for the development of anti-HCC therapies. As mentioned above, p53 and mTOR2 have exhibited their potential to modulate gluconeogenesis both through the regulation of PCKs $[9,10]$. However, the functions of these indicators are not restricted to the influence on glucose metabolism. Their complicated functions in diverse biological processes constrain their potent as targets for the development of anti-HCC therapies by modulating gluconeogenesis $[9,22-24]$.

For gluconeogenesis, the synthesis of PEP from OAA is an absolutely required process. Generally, this reaction strictly depends on the activity of PCK1 [25]. Mice with homozygous deletion of $P C K 1$ gene are impaired by severe hypoglycermia and profound changes in lipid and amino acid metabolism after birth [26]. Therefore, it was expected in the current study that the overexpression of $P C K 1$ would counteract the pro-survival function of metabolism reprogramming in HCC cells. The results of our experiments were promising in that PCK1 overexpression not only inhibited cell growth and migration in vitro but also restricted 
Fig. 6. Overexpression of PCK1 gene initiated the gluconeogenesis process in hep3B and SMMC-7721 cell lines. A, quantitative analysis results of RT-qPCR detection for G6PC gene with HCC cell samples. B, representative images and quantitative analysis results of western blotting assay for G6PC with HCC cell samples. C, quantitative analysis results of glucose production with HCC cell samples. “*”, significantly different from Control group, $\mathrm{P}<0.05$. "\#”, significantly different from NC group, $\mathrm{P}<0.05$. Each assay was represented by three replicates.
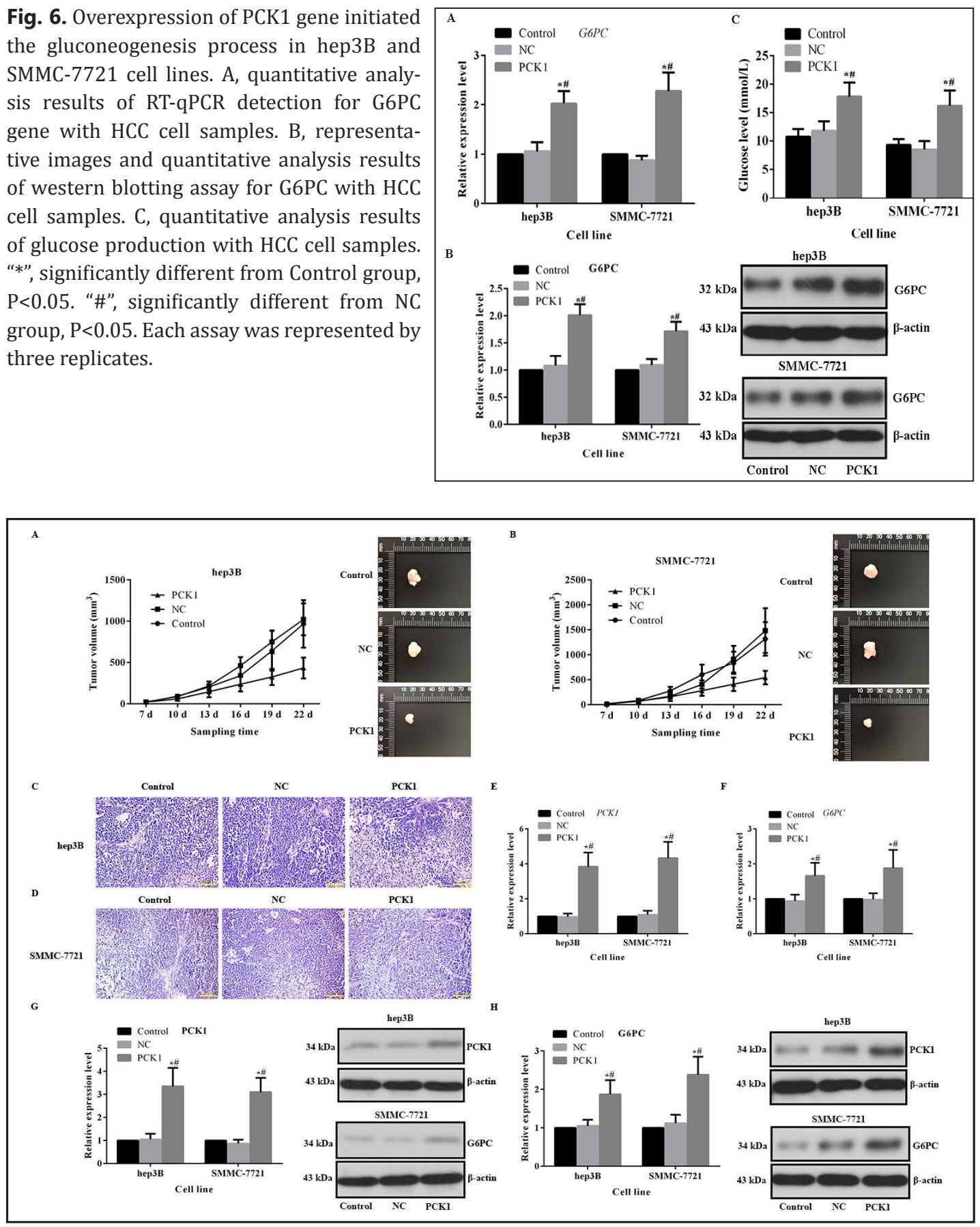

Fig. 7. Overexpression of PCK1 gene restricted growth of solid HCC and activated the gluconeogenesis process animals models. A, quantitative analysis result of tumor volume changes in animals transplanted with hep3B cells. B, quantitative analysis result of tumor volume changes in animals transplanted with SMMC7721 cells. C, representative images of $\mathrm{H} \& \mathrm{E}$ staining with tumor tissues in animals transplanted with hep3B cells. D, representative images of H\&E staining with tumor tissues in animals transplanted with SMMC-7721 cells. E, quantitative analysis results of RT-qPCR detection for PCK1 gene with tumor samples. F, quantitative analysis results of RT-qPCR detection for G6PC gene with tumor samples. G, representative images and quantitative analysis results of western blotting assay for PCK1 with tumor samples. H, representative images and quantitative analysis result of western blotting assay for G6PC with tumor samples. "*”, significantly different from Control group, $\mathrm{P}<0.05$. “\#”, significantly different from NC group, $\mathrm{P}<0.05$. Scale bar, 50 $\mu \mathrm{m}$. Each assay was represented by six replicates. 


\section{Cellular Physiology Cell Physiol Biochem 2018;47:344-355

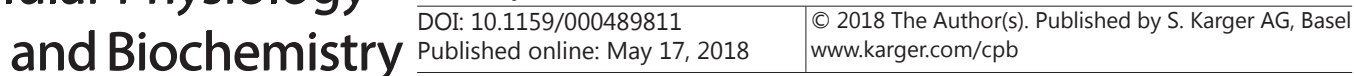 \\ Tang et al.: Activation of PCK1 Antagonizes HCC via Gluconeogenesis Initiation}

Table 1. Average tumor volume in mice transplanted with different HCC cell lines* - *Analyses based on ANOVA for repeated measurements showed that Group was an independent factor influencing the tumor volume. *, significantly different from Control group, $\mathrm{P}<0.05$. \#, significantly different from NC group, $\mathrm{P}<$ 0.05. Each assay was represented by six replicates

\begin{tabular}{|c|c|c|c|c|c|c|}
\hline \multirow{3}{*}{ Volume of tumor } & \multicolumn{6}{|c|}{ Cell Line } \\
\hline & \multicolumn{3}{|c|}{ hep3B } & \multicolumn{3}{|c|}{ SMMC-7721 } \\
\hline & Control & $\mathrm{NC}$ & PCK1 & Control & $\mathrm{NC}$ & PCK \\
\hline $7^{\text {th day }}$ & $18.59 \pm 4.7$ & $23.3 \pm 10.4$ & $21.6 \pm 8.1$ & $20.0 \pm 5.6$ & $14.2 \pm 1.9$ & $16.8 \pm 2.3$ \\
\hline $10^{\text {th }}$ day & $93.7 \pm 16.6$ & $88.7 \pm 14.4$ & $58.9 \pm 21.9^{* \#}$ & $83.7 \pm 39.3$ & $71.2 \pm 24.2$ & $70.4 \pm 40.9$ \\
\hline $13^{\text {th }}$ day & $204.7 \pm 65.0$ & $216.8 \pm 50.3$ & $147.1 \pm 68.1^{\star \#}$ & $278.5 \pm 81.4$ & $169.1 \pm 50.0$ & $159.7 \pm 83.9^{*}$ \\
\hline $16^{\text {th day }}$ & $340.1 \pm 121.9$ & $461.9 \pm 102.6$ & $238.6 \pm 87.7^{*}$ & $603.7 \pm 200.5$ & $406.3 \pm 80.4$ & $282.8 \pm 102.6^{* H}$ \\
\hline $19^{\text {th }}$ day & $642.1 \pm 239.4$ & $751.0 \pm 137.3$ & $325.3 \pm 98.0^{* *}$ & $838.5 \pm 223.9$ & $913.2 \pm 267.8$ & $410.7 \pm 133.8^{* \#}$ \\
\hline $22^{\text {nd day }}$ & $968.2 \pm 287.3$ & $1021.7 \pm 192.3$ & $434.5 \pm 126.3^{* \#}$ & $1318.7 \pm 335.0$ & $1484.8 \pm 447.3$ & $545.4 \pm 135.6^{* \#}$ \\
\hline
\end{tabular}

growth and invasiveness of solid HCC tumors in animal models without evident side effects on animal survival. To further determine the effect of PCK1 overexpression on glycolysis and gluconeogenesis, the levels of products and enzymes in glycolysis were measured. The results showed that in PCK1-overexpressed HCC cells, glycolysis process was evidently suppressed while gluconeogenesis process was initiated. The up-regulation of G6PC and PCK via mTOR2 inhibition induced the glucose production in cancer cells [10]. However, rather than increasing the mitochondrial oxidative phosphorylation, such redundant production of glucose only led to glucose consumption that was shunted off into gluconeogenesis [10]. Thus, the activation of PCK1 and G6PC in cancer cells created a "futile" cycle where glucose consumption was increased but only to have it to be broken down and then recycled through gluconeogenesis. Moreover, distinct from factors such as p53 and mTOR2, the function of $P C K 1$ is highly related to glucose metabolism especially in liver tissues [27]. The intended modulation of PCK1 gene has little influence on other biological processes, which can be employed as a highly specific candidate for the development of anti-HCC therapies.

In conclusion, the overexpression of PCK1 showed lethal effect on HCC cells. The induced expression of PCK1 not only inhibited cancer cell proliferation and migration in vitro but also restricted growth of solid tumors in vivo. Advantageous over the previous reported factors, the modulation of $P C K 1$ takes its action on the metabolism reprogramming feature of cancer cells and has little effect on other biological pathways. With more comprehensive studies in the future, the potential of $P C K 1$ as a highly specific and effective target for gluconeogenesisbased anti-HCC therapies will be fully explored.

\section{Acknowledgements}

The study was supported by National Natural Science Foundation of China (No. 81602571), Doctoral Scientific Research Foundation of Liaoning Province (No. 201603197), and Postdoctoral Science Foundation of China (No: 2015M572798).

\section{Disclosure Statement}

The authors disclose no conflict of interests.

\section{References}

1 Butler EB, Zhao Y, Munoz-Pinedo C, Lu J, Tan M: Stalling the engine of resistance: Targeting cancer metabolism to overcome therapeutic resistance. Cancer Res 2013;73:2709-2717.

-2 Khan MW, Chakrabarti P: Gluconeogenesis combats cancer: Opening new doors in cancer biology. Cell Death Dis 2015;6:e1872. 


\section{Cellular Physiology Cell Physiol Biochem 2018;47:344-355 \begin{tabular}{l|l} 
DOI: 10.1159/000489811 & $\begin{array}{l}\text { O 2018 The Author(s). Published by S. Karger AG, Basel } \\
\text { www.karger.com/cpb }\end{array}$
\end{tabular} \\ Tang et al.: Activation of PCK1 Antagonizes HCC via Gluconeogenesis Initiation}

3 Hanahan D, Weinberg RA: The hallmarks of cancer. Cell 2000;100:57-70.

4 Deberardinis RJ, Sayed N, Ditsworth D, Thompson CB: Brick by brick: Metabolism and tumor cell growth. Curr Opin Genet Dev 2008;18:54-61.

-5 Leithner K, Hrzenjak A, Trotzmuller M, Moustafa T, Kofeler HC, Wohlkoenig C, Stacher E, Lindenmann J, Harris AL, Olschewski A, Olschewski H: Pck2 activation mediates an adaptive response to glucose depletion in lung cancer. Oncogene 2015;34:1044-1050.

-6 Ward PS, Thompson CB: Metabolic reprogramming: A cancer hallmark even warburg did not anticipate. Cancer Cell 2012;21:10.

-7 Cairns RA, Harris IS, Mak TW: Regulation of cancer cell metabolism. Nat Rev Cancer 2011;11:85-95.

-8 Arai T, Kano F, Murata M: Translocation of forkhead box o1 to the nuclear periphery induces histone modifications that regulate transcriptional repression of pck1 in hepg2 cells. Genes Cells 2015;20:340-357.

-9 Goldstein I, Yizhak K, Madar S, Goldfinger N, Ruppin E, Rotter V: P53 promotes the expression of gluconeogenesisrelated genes and enhances hepatic glucose production. Cancer Metabol 2013;1.

10 Khan MW, Biswas D, Ghosh M, Mandloi S, Chakrabarti S, Chakrabarti P: Mtorc2 controls cancer cell survival by modulating gluconeogenesis. Cell Death Discovery 2015;1.

-11 Sheikh TI, Adam T, Qadri I: Upregulated hepatic expression of mitochondrial pepck triggers initial gluconeogenic reactions in the hcv-3 patients. Asian Pac J Trop Med 2015;8:618-623.

12 Bhalla K, Liu W-J, Thompson K, Anders L, Devarakonda S, Dewi R, Buckley S, Hwang B-J, Polster B, Dorsey SG: Cyclin d1 represses gluconeogenesis via inhibition of the transcriptional coactivator pgc1 $\alpha$. Diabetes 2014;63:3266-3278.

13 Ma R, Zhang W, Tang K, Zhang H, Zhang Y, Li D, Li Y, Xu P, Luo S, Cai W: Switch of glycolysis to gluconeogenesis by dexamethasone for treatment of hepatocarcinoma. Nat Commun 2013;4

14 Chang HC, Lane MD: The enzymatic carboxylation of phosphoenolpyruvate. Ii. Purification and properties of liver mitochondrial phosphoenolpyruvate carboxykinase. J Biol Chem 1966;241:2413-2420.

-15 Nordlie RC, Lardy HA: Mammalian liver phosphoneolpyruvate carboxykinase activities. J Biol Chem 1963;238:2259-2263.

16 Hanson RW, Garber AJ: Phosphoenolpyruvate carboxykinase. I. Its role in gluconeogenesis. Am J Clin Nutr 1972;25:1010-1021.

17 Kim H-J, Jee HJ, Yun J: DNA damage induces down-regulation of pepck and g6p gene expression through degradation of pgc-1 $\alpha$. Acta Bioch Bioph Sin 2011:gmr053.

18 Agirre M, Zarate J, Puras G, Ojeda E, Pedraz JL: Improving transfection efficiency of ultrapure oligochitosan/ DNA polyplexes by medium acidification. Drug Deliv 2014;22:100-110.

19 Shao J, Qiao L, Janssen RC, Pagliassotti M, Friedman JE: Chronic hyperglycemia enhances pepck gene expression and hepatocellular glucose production via elevated liver activating protein/liver inhibitory protein ratio. Diabetes 2005;54:976-984.

20 Gold J: Cancer cachexia and gluconeogenesis. Ann NY Acad Sci 1974;230:103-110.

21 Bongaerts GP, van Halteren HK, Verhagen CA, Wagener DJ: Cancer cachexia demonstrates the energetic impact of gluconeogenesis in human metabolism. Med Hypotheses 2006;67:1213-1222.

22 Sahin E, Colla S, Liesa M, Moslehi J, Muller FL, Guo M, Cooper M, Kotton D, Fabian AJ, Walkey C, Maser RS, Tonon G, Foerster F, Xiong R, Wang YA, Shukla SA, Jaskelioff M, Martin ES, Heffernan TP, Protopopov A, Ivanova E, Mahoney JE, Kost-Alimova M, Perry SR, Bronson R, Liao R, Mulligan R, Shirihai OS, Chin L, DePinho RA: Telomere dysfunction induces metabolic and mitochondrial compromise. Nature 2011;470:359-365.

23 Wang S-J, Yu G, Jiang L, Li T, Lin Q, Tang Y, Gu W: P53-dependent regulation of metabolic function through transcriptional activation of pantothenate kinase-1 gene. Cell Cycle 2013;12:753-761.

24 Sabatini DM: Mtor and cancer: Insights into a complex relationship. Nat Rev Cancer 2006;6:729-734.

25 Stark R, Guebre-Egziabher F, Zhao X, Feriod C, Dong J, Alves TC, Ioja S, Pongratz RL, Bhanot S, Roden M, Cline GW, Shulman GI, Kibbey RG: A role for mitochondrial phosphoenolpyruvate carboxykinase (pepck-m) in the regulation of hepatic gluconeogenesis. J Biol Chem 2014;289:7257-7263.

26 Hakimi P, Johnson MT, Yang J, Lepage DF, Conlon RA, Kalhan SC, Reshef L, Tilghman SM, Hanson RW: Phosphoenolpyruvate carboxykinase and the critical role of cataplerosis in the control of hepatic metabolism. Nutr Metab 2005;2:33.

27 Beale EG, Harvey BJ, Forest C: Pck1 and pck2 as candidate diabetes and obesity genes. Cell Biochem Bioph 2007;48:89-95. 